\title{
China's National Health Policies: An Ontological Analysis
}

\author{
Guobin Dai ${ }^{1}$, Fang Deng ${ }^{1}$, Arkalgud Ramaprasad ${ }^{2 *}$, Thant Syn ${ }^{3}$
}

1. Central South University, Changsha, Hunan, People Republic of China

2. University of Illinois at Chicago, Chicago, Illinois, United States of America

3. Texas A\&M International University, Laredo, Texas, United States of America

\section{Abstract}

The health care system in China is facing a multitude of challenges owing to the changing demographics of the country, the evolving economics of health care, and the emerging epidemiology of health as well as diseases. China's many national health care policies are documented in Chinese text documents. It is necessary to map the policies synoptically, systemically, and systematically to discover their emphases and biases, assess them, and modify them in the future. Using a logically constructed ontology of health care policies based on the common bodies of knowledge as a lens, we map the current policies to reveal their 'bright', 'light', and 'blind/blank' spots. The ontological map will help (a) develop a roadmap for future health care policies in China, and (b) compare and contrast China's health care policies with other countries'.

Keywords: National health care policy, ontology, text mapping, assessment, roadmap.

Correspondence: prasad@uic.edu*

DOI: $10.5210 /$ ojphi.v5i3.4933

Copyright @2016 the author(s)

This is an Open Access article. Authors own copyright of their articles appearing in the Online Journal of Public Health Informatics. Readers may copy articles without permission of the copyright owner(s), as long as the author and OJPHI are acknowledged in the copy and the copy is used for educational, not-for-profit purposes.

\section{Introduction}

The health care system in China is facing a multitude of challenges owing to the changing demographics and politics of the country, the evolving economics of health care, and the emerging epidemiology of health as well as diseases (1-4). For example, '[p]aralleled with the rapid socio-economic development and demographic transition, an epidemic of noncommunicable chronic diseases (NCDs) has emerged in China over the past three decades, resulting in increased disease and economic burdens... [and encouragement] to use communitybased health services.' (3) Between 1950-2009 it 'shifted public financing to private sources; it turned public hospitals and clinics into commercial, for-profit enterprises; it decentralized China's health system and it altered the price structure for public facilities, thereby enabling them to earn profits.' (1) In 2009, the 'Chinese government committed USD 124 billion of additional public spending for the first three years of health care reform.' (1) These three years had five specific targets. They were: '(1) expanding insurance coverage; (2) making public health service available and equal for all; (3) improving the primary care delivery system to 
provide basic health care; (4) establishing a national essential drug system; and (5) piloting public hospital reform.' (1)

Following the 2009 reform, five sets of comprehensive policies were introduced (5). They are summarized and quoted below as follows:

- Medical security: to achieve universal coverage through insurance systems for urban residents (urban employees' and residents' basic medical insurance), farmers (New Rural Cooperative Medical Service), and those living in poverty in both urban and rural areas (medical assistance).

- Public health services: full government funding for a minimum package of nine essential services (including maternal and child health, immunization, mental health, aged care, chronic disease management, and health education) with a view to equal access to public health services for all.

- Primary care: establishing a comprehensive network of basic health services through a three-tier rural network and urban community health services, with Chinese medicine integrated into these service delivery networks rather than as a freestanding system.

- Pharmaceutical supply system reform: setting an essential drug list that is linked to basic medical insurance and cooperative medical service, with open tender based on government reference price.

- Pilot hospital reforms: trialing strategies for ownership and government, quality, regulation, training, and revenue oversight.

These new policies did not completely eliminate the earlier polices. Thus, the post-2009 policies coexist with many pre-2009 policies. Given the number and complexity of these policies it would be difficult to obtain their 'big picture' from the corresponding text documents. Despite the best intentions of the policy makers, the complexity of the formulation process and the associated negotiations are likely to introduce differential emphases and biases (5-7). It is necessary to map the text of the policies systemically and systematically to discover their emphases and biases. Such an analysis will help the health policy-advisors and -makers formulate better policies. It can provide feedback on the current policies and feed-forward to future ones (8).

\section{Ontology of Health Care Policies}

The ontology of health care policy represents our conceptualization of the health care policy domain (9). It is an 'explicit specification of [our] conceptualization,' (10) and can be used to systematize the description of a complex system such as national health care policies (11). The ontology organizes the terminologies and taxonomies that constitute health care policies. 'Our acceptance of [the] ontology is... similar in principle to our acceptance of a scientific theory, say a system of physics; we adopt, at least insofar as we are reasonable, the simplest conceptual scheme into which the disordered fragments of raw experience can be fitted and arranged.' (12)

The method of constructing and presenting an ontology for a domain is an iterative process (13, 14) that systemically and systematically deconstructs health care policy into its dimensions and associated elements. It is a new method of analyzing and synthesizing knowledge in a domain 
$(15,16)$. It has been applied to analyzing and synthesizing Chile's and India's national health care policies $(17,18)$. Here we apply it to China's health care policies.

We deconstruct health care policy into (a) the scope of the policy, (b) the focus of the policy, (c) the desired outcomes of the policy, (d) the type of care which is the objects of the policy, and (e) the population for which it is intended. Thus, a health care policy is ideally seen as having a defined scope, being focused on an aspect of health care, with desired outcomes, for particular types of care, and targeted at a given population. Thus, the underlying argument is:

Health care policy = f (Scope, Focus, Outcome, Care, Population)

These five dimensions define the domain of health care policies. In practice, all health care policies may not cover all the dimensions. For example, some may not specify the outcome, some the focus, and some a combination of these. We will refer to policies in which some dimensions are excluded from the definition as fragments of policies rather than full policies.

Each dimension of the ontology is expressed by a taxonomy of its constituent elements (Figure 1). The dimensions and the elements are expressed both in English and Chinese. The elements are defined in the glossary in the Appendix. The taxonomies are derived from the common terminology in the body of knowledge on each dimension, especially in the health care policy domain. Thus, the Scope of a policy may be Global, National, Regional, Local-Urban, LocalRural, or restricted to the Provider. The Focus of the Policy may be Drugs, Food, Financial, Legal, Insurance, Technology, Information, Treatment, Personnel, or Administration. The Personnel focus may be on the Physician, Nurses, or Staff; the Physicians may be General or Specialist. The Outcome of the Policy may be Accessibility, Cost, Quality, Satisfaction, Safety, Parity, or Timeliness of health care. Further, the Care could be Preventive, for Illness (Mental, Physiological, Episodic, Chronic, or Occupational), Palliative, or Emergency. Last, the Population care for may be the Individual, Family, or Community. The Individuals may be Children, Adolescents, Adults (Female, Pregnant Woman, Worker, Disabled, Others), and Aged. (Note: We will capitalize the words which refer to the dimensions and elements in the ontology, except in describing full or partial components.) Symbolically:

Scope $\subset$ [Global, National, Regional, Local (Urban, Rural), Provider]

Focus $\subset$ [Drugs, Food, Financial, Legal, Insurance, Technology, Information, Treatment, Personnel (Physician (General, Specialist), Nurses, Staff), Administration]

Outcomes $\subset$ [Accessibility, Cost, Quality, Satisfaction, Safety, Parity, Timeliness]

Care $\subset$ [Preventive, Illness (Mental, Physiological, Episodic, Chronic, Occupational), Palliative, Emergency]

Population $\subset$ [Individual (Children, Adolescents, Adults (Female, Pregnant woman, Worker, Disabled, Others), Aged), Family, Community]

The ontology in Figure 1 helps visualize the dimensions, elements, and components of health care policy in natural English and Chinese. Each dimension is represented by a column. The taxonomy of elements constituting each dimension is listed in the respective column. All the 
elements together constitute the elements of the system of policies. The columns are connected by adjacent words/phrases - they help concatenate the elements from different columns into sentences which describe the components of the health care system.

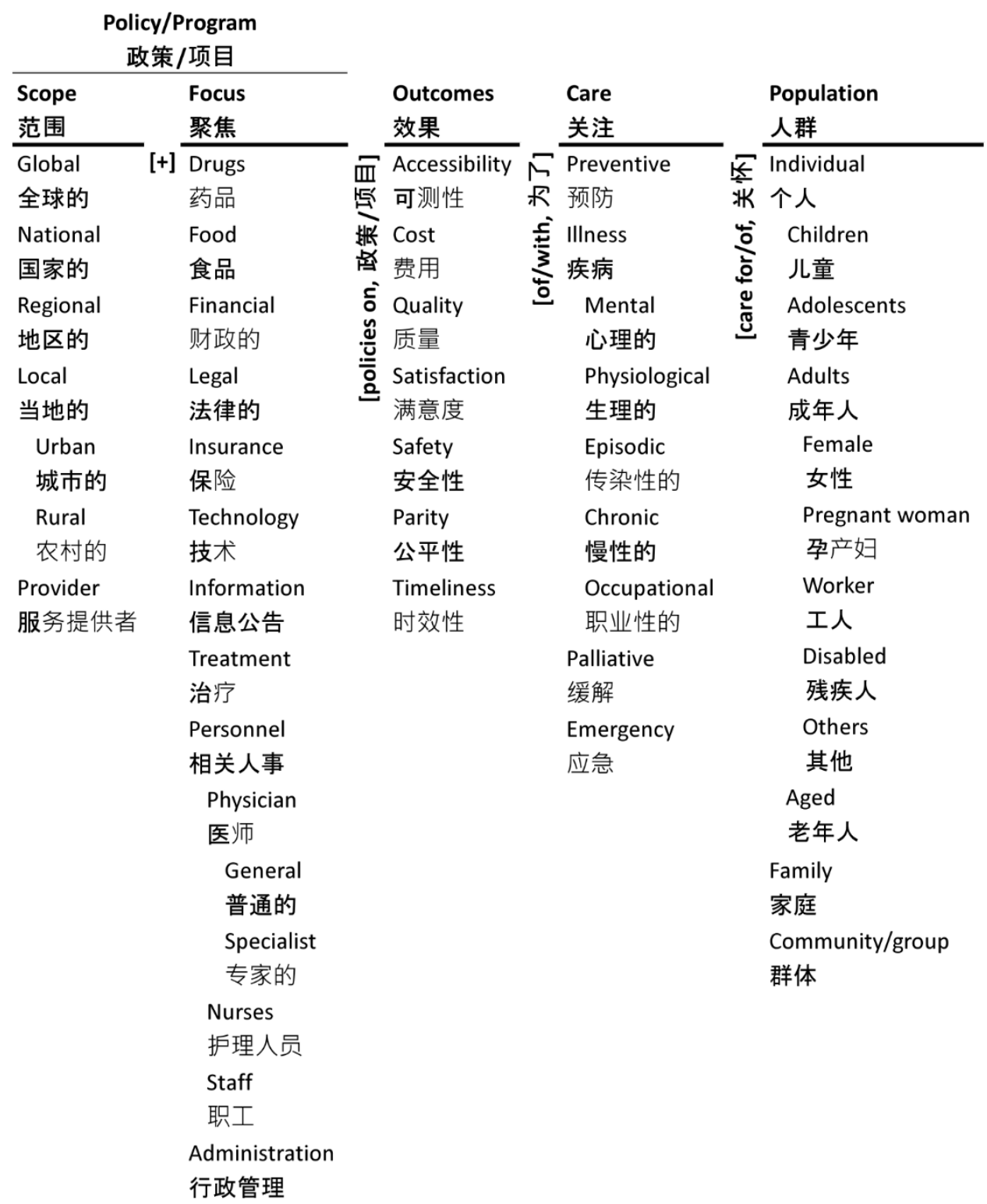

Illustrative components (total $=6 * 13 * 7 * 8 * 10=43,680$ ):

National financial policies on accessibility of preventive care for family. 国家财政政策针对提高家庭预防性护理的可测性

Local $_{\text {urban }}$ insurance policies on cost of palliative care for individual aged. 城市医保政策针对减少缓解老年人病痛的治疗费用

Provider administration policies on cost of illness episodic care of individual adolescents 服务提供者的行政管理政策针对减少青少年传染性疾病的治疗费用

Figure 1: Ontology of China’s Health Care Policy and Illustrative Components 
Three illustrative components derived from the ontology are shown in Figure 1. They are:

- National financial policies on accessibility of preventive care for family - these may include programs/policies to provide financial incentives for families to travel to obtain preventive care.

- Local urban regulatory policies on cost of palliative care for individual aged-these may include programs/policies to limit the cost of palliative care of senior citizens.

- Provider administration programs/policies on cost of illness episodic care of individual adolescents - these may include providers' policies on cost of care of ill teens.

These three and 43,677 others encapsulated in the ontology are logically the potential components of health care policies. The ontology presents the combinatorial complexity of health care policies concisely and thus helps us take a systemic view of the problems they address.

An element in the ontology may or may not be instantiated in a particular policy. Studying across policies, some of them may be instantiated frequently, some infrequently, and others not at all. We will label the frequently instantiated portions the 'bright' spots, the infrequent ones the 'light' spots, and the overlooked ones the 'blind/blank' spots.

The luminosity of each spot is a product of two opposing dynamics. A 'bright' spot may be so because it is effective and important; it may also be a consequence of habit and herd effect ('more of the same'), irrespective of whether it is effective or important. A 'light' spot may be so because it is ineffective and unimportant; it may also be a consequence of difficulty of implementing it ('path of least resistance'), irrespective of its potential effectiveness or importance, or its recent emergence in importance. A 'blind/blank' spot may have been simply overlooked by design or by accident; or, it may be infeasible.

Knowing the 'bright', 'light', and 'blind/blank' spots in the policies and their antecedent reasons will help develop more systemic and systematic approaches to the challenge of health care policies. In the following, we present an ontological map of China's health care policies, highlight the 'bright', 'light', and 'blind/blank' spots, and discuss possible reasons for the same. Before presenting the results, we will first describe the method we used for mapping. In the conclusion, we will present the potential implications of this program of research and the planned extensions to what is presented here.

The distinguishing feature of the proposed ontology is that it articulates the logic of the system in structured natural English and Chinese sentences. It is neither an abstract, philosophical discussion for meta-cognition (imagination) of the system, nor a concrete, syntactically precise formalization of the system for machine cognition. It is between the two extremes-a pragmatic, semantically rich articulation of the system for human cognition.

The ontology itself can be refined by adding subcategories of elements, coarsened by aggregating categories of elements, extended by adding additional dimensions, and reduced by eliminating current dimensions. Thus, it can be adapted to design health care policy at different levels of complexity and granularity $(13,15,19)$. 


\section{Validity of the Ontology}

The validity of the ontology will determine how well the ontology captures and represents China's health care policy. The common methods of validation focus on induced ontologies at a finer level of detail, greater formalism, and machine readability, but not on deduced ontologies at a higher-level of abstraction such as ours. Hence, we draw on the traditional constructs of validity and assert the ontology's face validity, content validity, semantic validity, systemic validity, and external validity, which are commonly used in social science research $(20,21)$.

The ontology is a complete and closed description of China's national health care policy. It is logically derived as described earlier. It is a novel reorganization and representation of traditional bodies of knowledge from multiple disciplines such as public policy, health care, and social science. It derives part of its validity from that of the knowledge in the underlying disciplines.

It comprehensively describes a country's health care policy using structured natural English and Chinese understandable to novices and experts alike. It deconstructs the combinatorial complexity of a construct and presents it visually as an easily readable, parsimonious text-table (22). The ontology encapsulates all 43,680 possible components of health care policy in a readable form on a single sheet of paper. Thus, its face validity is high.

Each logical component of national health care policy derived from the ontology is semantically meaningful in English and Chinese, as illustrated above-thus, its semantic validity is high. It is easy to ascertain whether a component is instantiated or not. Obversely, it is also easy to ascertain the component that matches an instantiation.

Its dimensions are based on the common bodies of knowledge from underlying disciplines. The taxonomies include the basic categories of knowledge from these disciplines. Hence, the content validity of the dimensions, the taxonomies, and the large number of consequent components is high.

Further, the ontology encapsulates all the possible components of China's national health care policy. It has high systemic validity.

Lastly, the ontology is also validated through application to the data (policies). The application will highlight errors of omission of significant elements and of commission of irrelevant elements in the ontology. These errors are easily corrected - the ontology is easily extensible and reducible - and the validity of the ontology enhanced. In the same vein, most of the ontology has been validated through application to Chile's and India's health care policies and learning from the same.

\section{Methods}

Two of the authors (who are Chinese) downloaded all the 289 health care policies in China from 2000-2016 which are currently in effect. These represent the population of policies post-2009 and currently operational policies pre-2009. The year 2009 represents a key turning point in the evolution of China's health care policies to achieve comprehensive universal health coverage by 2020 (2). The policies were downloaded from the website of the National Health and Phamiling Commission of the People's Republic of China 
(http://www.nhfpc.gov.cn/zhuzhan/index.shtml). Each policy has a separate address from which the current file was downloaded.

The distribution of policies by year is shown in Figure 2. One of the two authors is trained as a physician and is a faculty member; the other is a doctoral student. The former is very familiar with China's health care system. They downloaded all the policies as MS Word files (in Chinese) for coding. The two then coded half the policies (from the Chinese text) each onto the ontology using an Excel tool developed by another author. Subsequently, each of them verified the other's coding. Differences in interpretation were resolved through discussion. Thus, the final coding is the consensus of the two.

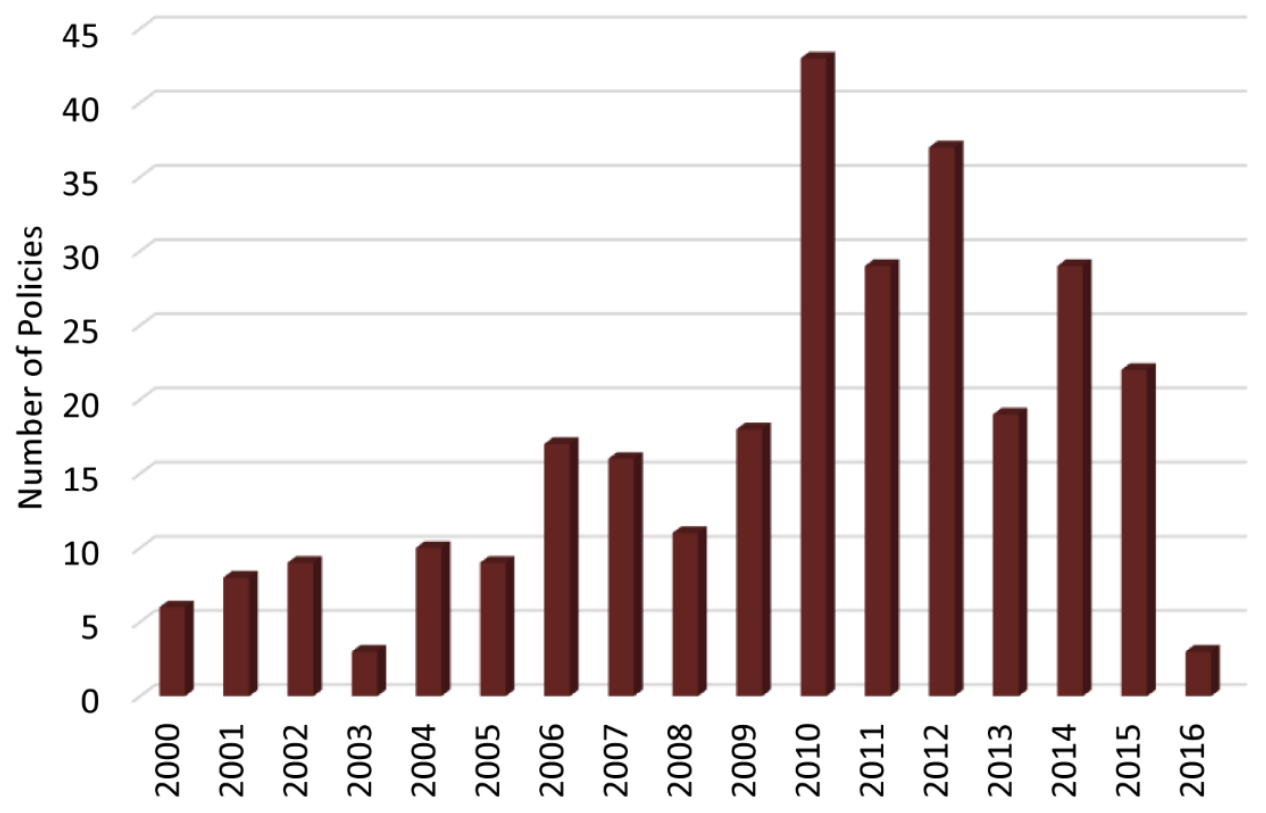

Figure 2: Distribution of China's Health Care Policies (Coded) by Year

A policy is coded on an item only if that item is explicitly part of it, not implied or inferred from it. For example, it may be argued that all National policies should be coded for Local-Urban and Local-Rural. But they were coded only for National unless the latter two are explicitly part of the policy. We sought to balance the errors of commission (over-coding) with the errors of omission (under-coding). The coders tried to balance over-reading the policy and thus over-coding, and under-reading the same and thus under-coding. The coders' knowledge of the domain-both Chinese, one a physician and faculty member, and the other a doctoral student-helped minimize the errors in coding.

We note that a program or policy may instantiate multiple components, a component, parts of multiple components, or part of a component of the ontology. Thus, there was no restriction on how many elements of the ontology could be encoded with reference to a policy, or a requirement that a policy should be encoded with reference to all the dimensions of the ontology. Thus, a policy could be encoded to:(a) an element from each dimension, (b) multiple elements from each dimension, (c) an element from some dimensions, or (d) multiple elements from some dimensions. We also note that the coding was binary — whether the element (or its synonym) was 
present or not in the program or policy. The coding was not weighted; each policy and each element was assigned equal weight.

\section{Results}

We present the results of the analysis as an ontological map (Figure 3). It highlights the emphasis on the elements of the ontology in the 289 policies. The number in parentheses adjacent to an element indicates its frequency of occurrence in the 289 policies. The data bar below the element is a visual indicator of the same scaled to the maximum frequency of occurrences of any one element, National (231) in this case. A policy may be coded on multiple elements on a dimension (column) or no items. Hence the sum of the numbers in each column may not equal 289-it may be more or less. The visualization in Figure 3 clearly highlights the areas of emphases, limited emphases, and no-emphasis. We will discuss the map in detail below from right to left.
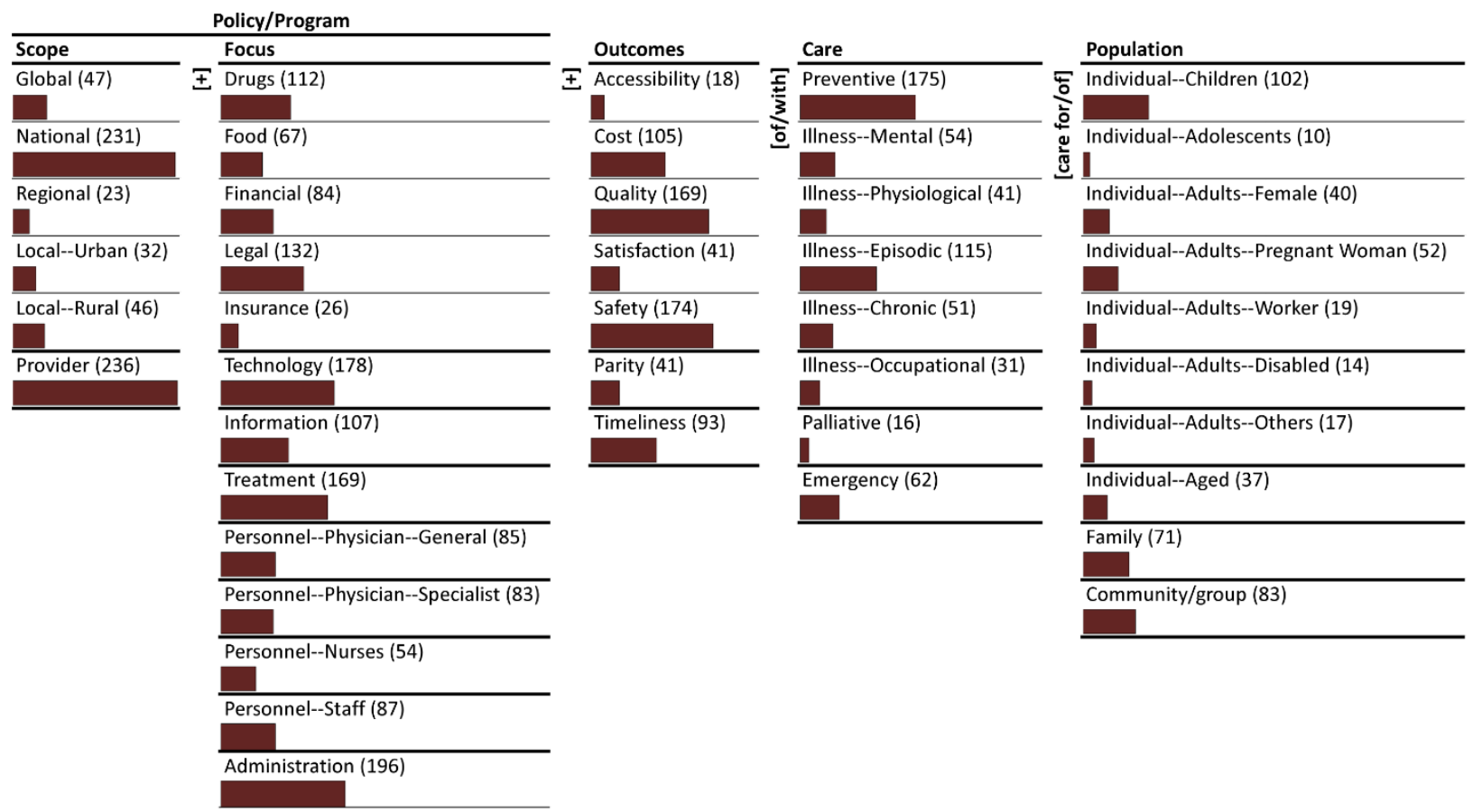

Figure 3: Ontological Map of Monads of China’s Health Care Policies

\section{Population}

Most of the policies focus on one or two segments of the population. The dominant focus of the policies is the Individual-Children (102), Community/Group (83), and Family (71). The policies are focused on children, communities, and families. The secondary focus of the policies is on Individual-Adults-Pregnant Woman (52), Individual-Adults-Female (40), and Individual-Aged (37). The tertiary focus is on Individual-Adults-Worker (19), Individual-Adults-Other (17), and Individual-Adolescents (10). It is noted, however, that there are 10 or more programs that address the needs of each segment of the population identified in the ontology. There are no 'blind/blank' spots in the Population dimension. 


\section{Care}

On average, each policy is focused on two types of care. The dominant focus of care is on Preventive (175) followed by Illness-Episodic (115). The secondary focus is on Emergency (62), Illness-Mental (54), Illness-Chronic (51), and Illness-Physiological (41) care. The tertiary focus is on Illness-Occupational (31) and Palliative (16) care. There is a minimum of 16 policies on each type of care; there are no 'blind/blank' spots in the Care dimension.

\section{Outcomes}

On average, each policy is focused on two or more types of outcome. The dominant outcomes sought are Safety (174) and Quality (169) of care. The secondary outcomes are Cost (105) and Timeliness (93) of care. Parity (41), Satisfaction (41), and Accessibility (18) are tertiary outcomes.

\section{Focus}

The policies, in the aggregate, are multi-focused - on average, more than four focuses per policy. The dominant focus is on Administration (196), Technology (169), Legal (132), Drugs (112), and Information (107). The secondary focus is on Personnel-Staff (87), Personnel-PhysicianGeneral (85), Financial (84), and Personnel-Physician-Specialist (83). The tertiary focus is on Food (67), Personnel-Nurses (54), and Insurance (26).

\section{Scope}

The focus of the policies is predominantly National (231) and on the Provider (236). The former is to be expected since the study is based on national policies. There is however, a secondary emphasis of these policies which is Global (47), Local-Rural (46), Local-Urban (32), and Regional (23).

\section{Ontological Map Summary}

In summary, the dominant focus of the policies based on the ontological map may be summarized as follows:

Provider/National Administration/Technology/Legal/Drugs/Information policies on Safety/Quality of Preventive/Illness-Episodic care of Individual-Children/ CommunityGroup/Family.

\section{Discussion}

The five focus of China's health care policy reform are: (a) medical security through insurance, (b) comprehensive public health services, (c) basic primary care, (d) supply system for essential drugs, and (e) hospital reforms $(1,5)$. We will discuss our results from the ontological map with reference to these focuses.

The relatively low emphasis on Insurance (26) among the 289 policies runs contrary to the first focus. It may be partly because China has already achieved universal insurance coverage (2). 
Despite the coverage there are many significant issues in translating it into significant health outcomes-for example, in the managing non-communicable chronic diseases (3) and access to rural health care (23). The results above suggest the need for a greater focus on insurance in conjunction with different combinations of outcomes and types of care.

The emphasis on public health is evident in the dominance of Community/Group (83) and Family (71). However, relatively less emphasis on Illness-Chronic (51) appears to limit the comprehensiveness of the care, especially in light of the growing importance of chronic disease management in China $(3,24)$.

The heavy emphasis on Individual-Children (102), Community/Group (83) and Family (71) combined with that on Preventive (175) and Illness-Episodic (115) can be said to indicate the focus on basic primary care. However, the limited emphasis on Accessibility (18) and IllnessChronic may significantly weaken the delivery of such care.

The dominance of policies on Drugs (112) is likely a manifestation of the emphasis on the supply system for essential drugs. It appears to be closely associated with Quality (169) and Safety (174) of Illness-Episodic (115) and Preventive (175) care. It also appears to be closely associated with Treatment (169), Legal (132), Administration (196), and Provider (236) policies. It is an important but complex issue (25-28) and the policies seek to address the same.

There is probably no better indicator of the emphasis on hospital reforms than the priority given to Providers (236) and Administration (196). The two appear to be dominant in conjunction with Technology (178), Information (107), and other policies. Together, they can be seen to be part of a recipe for transforming the hospitals.

In summary, the results from the ontological map of monads highlight the alignment, mis-alignment, and non-alignment of the 289 policies with the five focuses of the 2009 health care reforms in China. The alignments have to be reinforced, the mis-alignments redirected, and non-alignments rectified in the future for the transformation to be effective.

\section{Conclusions}

The challenge of reflecting upon China's national health care policies and reorienting them is one of synthesizing a large body of text-based knowledge in Chinese systemically and systematically. It is a knowledge management problem and a 'big-text-data' analysis problem. The ontology of China's health care policies is comprehensive lens for the analysis and synthesis of this corpus in the original language. It can be used to develop a roadmap for future research on China's health care policies.

The paper makes three basic contributions to understanding, comprehensive assessment, and direction of China's health care policies. They are:

- An approach using an ontology based on national health care policy. The approach can be extended, refined, revised, and adapted to other contexts (provinces, cities, etc.); 
- A method of mapping the national health care policies onto the ontology based on the original text of the policies (in this case Chinese); and

- A method of analyzing the dominant, less dominant and non-dominant focus of a nation's policies, and the consequent gaps in them.

China's health care policies have undergone waves of reform (4). In making these reforms there is a need for policy makers to understand the implications of the above findings for future planning, and prioritizing for formulation (and reformulation) of policy. Our research will allow the policy makers to ask, as discussed above, for example: Are the dominant foci e.g. preventative care, illness-episodic care, quality, and safety balanced against the low focus elements such as illness-chronic care, palliative care, and accessibility? Do they need to be? Ultimately ontological analysis such as this can provide a pragmatic basis for deliberations (7) by policy makers and interest groups for policy formulation.

The framework, the method, and the results can be useful to advance health policy making in general and China's health policies in particular. It can be used to assess the strengths and weaknesses of a country's health policy, and to compare and contrast policies of different countries based on a common framework. The present study is a continuation of our study of Chile, India, and Australia's healthcare policies and programs. Understanding the antecedents and consequences of the emphases in other countries can provide insights into the policies of the parent country.

In ending the discussion, we should also highlight some of the limitations of the research. The ontology may be incomplete or over-specified. In the future, should it be necessary, the ontology can be extended, reduced, refined, or coarsened as appropriate.

Considerable effort has been expended in the translation of the ontology, construction of the glossary, and the monitoring of coding to minimize errors. While the coders tried to stay true to the text of the policies without imputing their own expectations, one cannot exclude the possibility of over-coding and under-coding. Given the large population of programs (289) and the significant variation in the frequency of the elements, despite the potential errors, the results are likely to be robust.

Given the data, errors in the ontological map are unlikely. However, there is room for variation in the interpretation of the luminosity of the different elements. Since the method of construction of the map is completely transparent, it would be easy to compare and contrast different interpretations of the same.

In summary, despite the limitations, the insights are strong. Their explanation as to why the corpus is as described may vary, but there is little room for variation in the description of the policies corpus.

\section{Financial Disclosure}

This study is partially supported by the Chinese National Social Science Fund 15BGL104 and Chinese Ministry of Education of Humanities and Social Science Youth Project 12YJC630028. The views represented in this article are those of the individual authors only. 


\section{References}

1. Hsiao WC, Li M, Zhang S. Universal Health Coverage: the Case of China. 2013.

2. Yip WC, Hsiao WC, Chen W, Hu S, Ma J, et al. 2012. Early appraisal of China's huge and complex health-care reforms. Lancet. 379(9818), 833-42. PubMed http://dx.doi.org/10.1016/S0140$\underline{6736(11) 61880-1}$

3. Xiao N, Long Q, Tang X, Tang S. 2014. A community-based approach to non-communicable chronic disease management within a context of advancing universal health coverage in China: progress and challenges. BMC Public Health. 14(2), 1-6. doi:10.1186/1471-2458-14-s2-s2. PubMed

4. Yip WC, Hsiao WC. 2015. What Drove the Cycles of Chinese Health System Reforms? Health Syst Reform. 1(1), 52-61. doi:http://dx.doi.org/10.4161/23288604.2014.995005.

5. Lin V, Zhao H. Health Policy Reform in China. In: E. Kuhlmann et al., editor. The Palgrave International Handbook of Healthcare Policy and Governance: Palgrave Macmillan; 2015.

6. Kornreich Y, Vertinsky I, Potter PB. 2012. Consultation and Deliberation in China: The Making of China's Health-Care Reform. China J. (68), 176-203. doi:http://dx.doi.org/10.1086/666583.

7. Korolev A. 2014. Deliberative Democracy Nationwide?-Evaluating Deliberativeness of Healthcare Reform in China. J Chinese Polit Sci. 19(2), 151-72. doi:http://dx.doi.org/10.1007/s11366-014-9287$\underline{1 .}$

8. Ramaprasad A. On the Definition of Feedback. Behav Sci. 1983;28(1):4-13. doi: 10.1002/bs.3830280103. PubMed PMID: ISI:A1983QA64700002.

9. Gruber TR. Ontology. In: Liu L, Özsu MT, editors. Encyclopedia of Database Systems. New York: Springer-Verlag; 2008.

10. Gruber TR. 1995. Toward Principles for the Design of Ontologies Used for Knowledge Sharing. Int J Hum Comput Stud. 43(5-6), 907-28. doi:http://dx.doi.org/10.1006/ijhc.1995.1081.

11. Cimino JJ. 2006. In defense of the Desiderata. J Biomed Inform. 39(3), 299-306. doi:http://dx.doi.org/10.1016/j.jbi.2005.11.008. PubMed

12. Quine WVO. From a Logical Point of View. Second, revised ed. Boston, MA, USA: Harvard University Press; 1961.

13. Ramaprasad A, Syn T. Design Thinking and Evaluation Using an Ontology. In: Helfert M, Donnellan B, Kenneally J, editors. Design Science: Perspectives from Europe. Communications in Computer and Information Science. 447. Switzerland: Springer International Publishing; 2014. p. 63-74.

14. Ramaprasad A, Syn T. Ontological Topography: Mapping the Bright, Light, Blind/Blank Spots in Healthcare Knowledge. Proceedings of the 2nd International Conference on Big Data and Analytics in Healthcare (BDAH 2014). Singapore2014. 
15. Ramaprasad A, Syn T. 2015. Ontological Meta-Analysis and Synthesis. Comm Assoc Inform Syst. 37, 138-53.

16. Tate M, Furtmueller E, Evermann J, Bandara W. 2015. Introduction to the Special Issue: The Literature Review in Information Systems. Comm Assoc Inform Syst. 37, 103-11.

17. Núñez Mondaca A, Ramaprasad A, Syn T. National Healthcare Policies in Chile: An Ontological Meta-Analysis. In: Sarkar IN, Georgiou A, Mazzoncini de Azevedo Marques P, editors. MEDINFO 2015: eHealth-enabled Health. Studies in Health Technology and Informatics. 2162015. p. 1105.

18. Ramaprasad A, Sastry NKB, Syn T. National Healthcare Programs and Policies in India: An Ontological Analysis. Proceedings of the 10th Annual International Conference on Public Policy and Management (10 CPPM 2015). Bangalore, India2015.

19. Ramaprasad A, Syn T, Win KT. Ontological Meta-Analysis and Synthesis of HIPAA. Proceedings of the 18th Pacific Asia Conference on Information Systems (PACIS 2014). Chengdu, China2014.

20. Brennan L, Voros J, Brady E. 2011. Paradigms at play and implications for validity in social marketing research. J Soc Market. 1(2), 100-19. doi:http://dx.doi.org/10.1108/20426761111141869.

21. Horn BR, Lee I-H. Toward integrated interdisciplinary information and communication sciences: a general systems perspective. Proceedings of the 22nd Hawaii International Conference on System Sciences (HICSS 1989). 4. Kailua-Kona, Hawaii, USA: IEEE; 1989. p. 244-55.

22. Tufte ER. Envisioining Information. Cheshire, CT: Graphics Press; 1990.

23. Liu X, Tang S, Yu B, Phuong NK, Yan F, et al. 2012. Can rural health insurance improve equity in health care utilization? a comparison between China and Vietnam. Int J Equity Health. 11(1), 1-9. doi:http://dx.doi.org/10.1186/1475-9276-11-10. PubMed

24. Li T, Lei T, Xie Z, Zhang T. 2016. Determinants of basic public health services provision by village doctors in China: using non-communicable diseases management as an example. BMC Health Serv Res. 16(1), 1-10. doi:10.1186/s12913-016-1276-y. PubMed

25. Ou Y, Jing B-q, Guo F-f, Zhao L, Xie Q, Fang Y-l, et al. 2014. Audits of the quality of perioperative antibiotic prophylaxis in Shandong Province, China, 2006 to 2011. Am J Infect Control. 42(5), 516-20. doi:http://dx.doi.org/10.1016/j.ajic.2014.01.001. PubMed

26. Tang Y, Zhang X, Yang C, Yang L, Wang H, et al. 2013. Application of propensity scores to estimate the association between government subsidy and injection use in primary health care institutions in China. BMC Health Serv Res. 13(1), 1-7. doi:http://dx.doi.org/10.1186/1472-6963-13-183. PubMed

27. Yang L, Liu C, Ferrier JA, Zhou W, Zhang X. The impact of the National Essential Medicines Policy on prescribing behaviours in primary care facilities in Hubei province of China. Health Pol Plann. 2012:czs116. doi: 10.1093/heapol/czs116. 
28. Li Y, Xu J, Wang F, Wang B, Liu L, et al. 2012. Overprescribing In China, Driven By Financial Incentives, Results In Very High Use Of Antibiotics, Injections, And Corticosteroids. Health Aff. 31(5), 1075-82. doi:http://dx.doi.org/10.1377/hlthaff.2010.0965. PubMed

\section{Appendix: Glossary}

Policy: Health care policy.

Scope: Reach of the health care policy.

Global: Policy applicable in all countries of the world.

National: Policy applicable everywhere in China.

Regional: Policy applicable to a region of China.

Local: Policy applicable within a defined part of China.

Urban: Policy applicable within local urban areas.

Rural: Policy applicable within local rural areas.

Provider: Policy applicable to a health care providing institution.

Focus: Focus of the health care policy.

Drugs: Policies regarding drugs used in health care.

Food: Policies regarding food and nutrition in health care.

Financial: Policies regarding health care finance.

Legal: Policies regarding legal issues in health care.

Insurance: Policies regarding health care insurance.

Technology: Policies regarding health care technology.

Information: Policies regarding health care information.

Treatment: Policies regarding treatment.

Personnel: Policies regarding health care personnel.

Physician: Policies regarding physicians.

General: Policies regarding general physicians.

Specialist: Policies regarding specialist physicians. 
Nurses: Policies regarding health care nurses.

Staff: Policies regarding health care staff.

Administration: Policies regarding health care administration.

Outcomes: The intended outcomes of health care policy.

Accessibility: The accessibility of health care to the population.

Cost: The cost of health care to the population.

Quality: The quality of health care delivered to the population.

Satisfaction: The population's satisfaction with health care.

Safety: The safety of health care delivered to the population.

Parity: The parity of health care delivered to the population segments.

Timeliness: The timeliness of health care delivery to the population.

Care: The different types of health care.

Preventive: Care to prevent illnesses and diseases in the population.

Illness: Care of illnesses when they occur.

Mental: Care of mental illness.

Physiological: Treatment of physiological illness

Episodic: Care during illness episodes -- time bound.

Chronic: Care of chronic illnesses -- continuing.

Occupational: Treatment of occupational illness.

Palliative: Care to alleviate pain and suffering.

Emergency: Care of emergency illness

Population: The population targeted by the policy.

Individual: Individual recipients of health care.

Children: Children who are recipients of health care.

Adolescents: Adolescents receiving health care. 
Adults: Adults receiving health care.

Female: Women receiving health care.

Pregnant women: Pregnant women receiving maternal health care.

Workers: Workers receiving occupational/work related health care.

Disabled: Disabled adults receiving health care.

Other: Adults other than mothers and workers.

Aged: Older people receiving elderly health care.

Family: Family, as an entity, receiving health care.

Community/group: Community/group as an entity receiving health care. 